\title{
The Risk For Malignancy of the Thyroid Nodule is Modulated by Gender, Echotexture, and Intranodular Lymphocytic Thyroiditis
}

Authors

Salvatore Arena', Salvatore Benvenga2, 3, 4

Affiliations

1 A.S.P. 8 Siracusa, Section of Endocrinology and Metabolic Diseases, Department of Internal Medicine, Umberto I Hospital, Siracusa, Italy

2 Department of Clinical and Experimental Medicine, University of Messina, Messina, Italy

3 Interdepartmental Program of Molecular \& Clinical Endocrinology, and Women's Endocrine Health, AOU Policlinico G. Martino, Messina, Italy

4 Master Program on Childhood, Adolescent and Women's Endocrine Health, University of Messina, Messina, Italy

Key words chronic lymphocytic thyroiditis, thyroid cancer, thyroid fine-needle aspiration cytology, thyroid ultrasonography

$$
\text { received } \quad 16.02 .2019
$$

accepted 06.08.2019

\section{Bibliography}

DOI https://doi.org/10.1055/a-0994-0489

Horm Metab Res 2019; 51: 559-567

(c) Georg Thieme Verlag KG Stuttgart · New York

ISSN 0018-5043

Correspondence

Salvatore Arena, MD

Endocrinologia e Diabetologia

Azienda Sanitaria Provinciale 8, Siracusa

Presidio Ospedaliero Umberto I

Via Testaferrata 1

96100 Siracusa

Italy

Tel.: + 39/0931/724 098; Mobile.: + 39/338/462 9779

Fax: + 39/0931/724 100

salvoarena72@hotmail.com; arenaendo@gmail.com

\section{ABSTRACT}

Based on the American (Bethesda, 2017) or Italian (SIAPEC 2014) cytological categories of thyroid nodules, the risk of malignancy and management vary. This risk is $5-10 \%$ or $<3 \%$ (benign or TIR2), 6-18\% or $<10 \%$ (AUS/FLUS or TIR3A), $10-40 \%$ or $15-30 \%$ (FN/SFN or TIR3B), $45-60 \%$ or $60-80 \%$ (suspicious or TIR4), $94-96 \%$ or $95 \%$ (malignant or TIR5). In 408 thyroid nodules evaluated cytologically, we computed the malignancy rate in each category considering gender (325 females, 83 males), echotexture (268 isoechoic, 140 hypoechoic), intranodular chronic lymphocytic thyroiditis (ICLT: 113 with and 295 without); histology (263 benign, 145 malignant). It was $0-1.7 \%$ for the benign categories, except hypoechoic/ICLT + ve nodules of females (25\%); 0-2.3\% for the AUS/FLUS category, except isoechoic/ICLT-ve nodules of males (11.1\%) and hypoechoic/ ICLT-ve nodules of females (22.2\%). For the FN/SFN category, rate was the most variable (from $0 \%$ in isoechoic/ICLT + ve nodules of males to $100 \%$ in hypoechoic/ICLT-ve nodules of males). The $30 \%$ threshold for risk was passed in four subgroups, and the $40 \%$ threshold in two subgroups ( $45 \%$ in isoechoic/ICLT-ve nodules of males, $80 \%$ in hypoechoic/ICLT + ve nodules of females). For the suspicious category, rate was $100 \%$ in males, except those with isoechoic/ICLT-ve nodules (75\%), and $>80 \%$ in females with hypoechoic nodules. For the malignant category, rate was always $100 \%$. In conclusion, particular groups of nodules (based on gender, echotexture, and ICLT) within the cytologically benign through the suspiciously malignant category are at risk of malignancy substantially greater (even 100\%) than the standard one. Accordingly, the suggested management cannot be standardized.

\section{Introduction}

Thyroid ultrasonography (US) has been recognized as an important step for selecting nodules that are worthy of cytological investigation by fine-needle aspiration (FNA). In turn, FNA diagnostic categories are important for the decision-making process about their management, since the associated risk for malignancy varies in each category [ 1,2$]$. The 6 -tier categorization of the cytological di- agnosis of thyroid nodules, according to both the Italian system [3] and the American system [4,5], with the corresponding risks for thyroid malignancy, is illustrated in $>$ Table 1 . The recently updated Bethesda system [5] provides different risks, depending upon the fact that a recently defined tumor entity (noninvasive follicular thyroid neoplasm with papillary-like nuclear features [NIFTP]) [6] is considered a benign or a malignant tumor. 
In our clinical practice on thyroidectomized patients, we have noticed that when the presence of lymphocytic infiltration of the FNA-interrogated nodule had been mentioned in the cytological report, this had a different predictive value for malignancy depending on echotexture and gender [7]. For instance, nodules containing a lymphocytic infiltrate, having the same echotexture (hypoechogenicity) and belonging to the same FNA category (follicular neoplasm [FN] or suspicious for a follicular neoplasm [SFN]), had a relatively low risk of malignancy if the patient was male, but a very high one if the patient was female [7]. As well known, the detection of lymphocytic infiltration in the FNA-interrogated thyroid nodule (see below, Patients and Methods) and the ensuing diagnosis of chronic lymphocytic thyroiditis is just used to categorize the nodule as benign if there are no other associated cytological signs of malignancy.

Hence, we retrospectively reviewed a consecutive series of patients residing in the same geographical area who underwent both FNA and thyroidectomy, and computed the frequency of malignancy for each FNA category. Such frequency was computed also in subgroups that were formed based on echotexture (hypoechogenicity or isoechogenicity), status for intranodular lymphocytic thyroiditis (presence or absence) and gender (male or female).

\section{Patients and Methods}

Our study relies upon the fact that all US and FNA evaluations (including cytological reading) were performed by the same operator (S.A.), who routinely looks for the absence or presence of ICLT. The first patient in our cohort of 408 patients underwent US-assisted FNA in 2010, well before the current ATA guidelines published in January 2016 [2], and the last patient did so in 2016. The guidelines used to select nodules worthy of FNA interrogation were those released by the ATA in November 2009 [8], according to which the recommended nodule threshold size was $>10 \mathrm{~mm}$ if hypoechoic, $\geq 10-15 \mathrm{~mm}$ if isoechoic or hyperechoic, or $>5 \mathrm{~mm}$ for high-risk history nodules regardless of echotexture. According to the most recent ATA guidelines [2], FNA for hypoechoic nodules is recommended if the maximum diameter is $\geq 10 \mathrm{~mm}$, while FNA for isoechoic or hyperechoic nodules is recommended if the maximum diameter is $\geq 15 \mathrm{~mm}$.

To form our said cohort, we considered solid nodules that were categorized based on echotexture (isoechoic or hypoechoic), cytological classes of risk ( $\vee$ Table 1 ), cytological detection (presence or absence) of ICLT, histological diagnosis (benign or malignant) and gender (males or females). Cystic nodules as well as cytologically nondiagnostic nodules were excluded. As reported previously [9-12] the presence of ICLT was based on detection of diffuse presence of lymphocytes and germinal centers in the background and/or infiltrating thyroid follicles with marked signs of inflammation and moderate amounts colloid. Additional finding that could or could not be present were follicular atrophy, plasma cells, multinucleated giant cells, epithelioid cell clusters, intralobular fibrosis, and Hurtle cell metaplasia. Such metaplasia is characterized by nuclear grooves, chromatin clearing, prominent nucleoli, increased nuclear size, irregular nuclear shapes and nuclear atypia, sometimes overlapping with malignant lesions.

\section{Statistics}

We compared continuous variables using the two-tailed Student's t-test, and compared categorical variables using the chi-square test $\left(\mathrm{X}^{2}\right)$ or Fisher's exact test, as appropriate.

\section{Results}

In order to permit a synoptic view, cytological data are presented

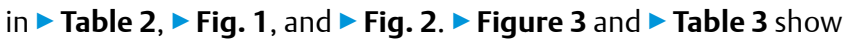
data with the fundamental finding of different risks for malignancy within the same cytological category upon taking into account ultrasound nodular pattern, ICLT status and gender.

- Table 1 Cytological categorization of thyroid nodules according to the specified systems, and associated risk of malignancy in each category.

\section{Cytological category}

\begin{tabular}{|l|}
\hline Bethesda 2007 and 2017 \\
\hline
\end{tabular}

Nondiagnostic or unsatisfactory

Benign

Atypia of undetermined significance or follicular lesion of undetermined significance

Follicular neoplasm or suspiciousfor a follicular neoplasm

Suspicious for malignancy

Malignant
SIAPEC 2014

$$
\text { Bethesda } 2007
$$

Bethesda, 2017

Risk of Malignancy, \%

NIFTP: Noninvasive follicular thyroid neoplasm with papillary-like nuclear features; CA: Carcinoma. 


\section{Distribution of the five FNA classes based on gender, echotexture, and ICLT}

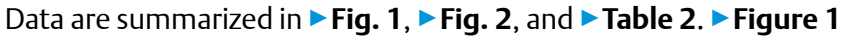
shows the distribution of nodules across the cytological classes of risk based on gender (top panel), echotexture (middle panel) and ICLT status (bottom panel). Males were overrepresented in the TIR3B category [ 27 of all 83 males (32.5\%; $>$ Fig. 1 ), and 27 of all 98 nodules in this category $(27.6 \%$; $>$ Fig. 2$)$ ] while females were overrepresented in the TIR2 category [ 96 of all 325 females (29.5\%; - Fig. 1), and 96 of the 111 nodules in this category (86.5\%; - Fig. 2)]. Hypoechoic nodules were overrepresented in the TIR4 category [44 of all 140 hypoechoic nodules (31.4\%; - Fig. 1), and 44 of all 70 nodules in this category (62.9\%; $>$ Fig. 2$)$ ], while isoechoic nodules were overrepresented in the TIR2 category [88 of all 268 isoechoic nodules (32.8\%; - Fig. 1), and 88 of all 111 nodules in this category (79.3\%; $>$ Fig. 2)]. Similarly, ICLT + ve nodules were overrepresented in the TIR4 category [34 of all 113 ICLT + ve nodules (30.1\%; $>$ Fig. 1), and 34 of all 70 nodules in this category
(48.6\%; > Fig. 2)], while ICLT-ve nodules were overrepresented in the TIR2 category [ 86 of all 295 ICLT-ve nodules (29.1\%; $\triangleright$ Fig. 1), and 86 of all 111 nodules in this category (77.5\%; $>$ Fig. 2)]. Overall, the distribution of the five cytological classes between males and females did not reach statistical significance $(p=0.11$; $\triangleright$ Fig. 1, top panel). From $>$ Fig. 2 (top panel), it can be inferred that the female to male ratio was the highest in the TIR2 nodules and the lowest in the TIR3B nodules (F:M $=6.4$ and 2.6, respectively).

In contrast, the distribution differed significantly between the hypoechoic and the isoechoic nodules $\left(p=7.4 \times 10^{-9}\right.$; $>$ Fig. 1 , middle panel), with an over-representation of the TIR4 and TIR5 classes in the hypoechoic group [44/140 (31.4\%) vs. 26/268 (9.7\%), $X^{2}=30.5, p=3.3 \times 10^{-8}, O R=4.3(2.5-7.3)$, and $22 / 140(15.7 \%) v s$. $\left.21 / 268(7.8 \%), X^{2}=6.05, p=0.014, O R=2.2(1.2-4.1)\right]$. In contrast, the TIR2 class was overrepresented in the isoechoic group [88/268 (32.9\%) vs. $23 / 140$ (16.5\%), $\left.\left.X^{2}=12.50, p=0.0004, O R=2.59\right)\right]$. - Figure 2 (middle panel) shows that hypoechoic nodules are less

- Table 2 Distribution of the cytological categories based on the given indices.

\begin{tabular}{|c|c|c|c|c|c|c|c|}
\hline & No. & TIR2 & TIR3A & TIR3B & TIR4 & TIR5 & Statistics \\
\hline All & 408 & $111(27.2)$ & $86(21.1)$ & $98(24.0)$ & $70(17.2)$ & $43(10.5)$ & $\mathrm{N} / \mathrm{A}$ \\
\hline \multicolumn{8}{|l|}{ Echotexture and sex } \\
\hline Hypoechoic, M & 24 & $0^{\mathrm{b}}$ & $7(29.2)^{b}$ & $6(25.0)$ & $6(25.0)$ & $5(20.8)$ & \multirow{2}{*}{$\begin{array}{l}X^{2}=9.22 \\
p=0.055\end{array}$} \\
\hline $\mathrm{F}$ & 116 & $23(19.8)^{b}$ & $15(\mathbf{1 2 . 9})^{\mathrm{b}}$ & $23(19.8)$ & $38(32.8)$ & $17(14.7)$ & \\
\hline Isoechoic, M & 59 & $15(25.4)$ & $11(18.6)$ & $21(35.6)^{\mathrm{a}}$ & $6(10.2)$ & $6(10.2)$ & \multirow{2}{*}{$\begin{array}{l}x^{2}=5.53 \\
p=0.24\end{array}$} \\
\hline $\mathrm{F}$ & 209 & $73(34.9)$ & $53(25.3)$ & $48(23.0)^{\mathrm{a}}$ & $20(9.6)$ & $15(7.2)$ & \\
\hline \multicolumn{8}{|l|}{ ICLT and sex } \\
\hline ICLT + ve, M & 16 & $2(12.5)$ & $4(25.0)$ & $5(31.3)$ & $3(18.7)$ & $2(12.5)$ & \multirow{2}{*}{$\begin{array}{l}x^{2}=3.51 \\
p=0.48\end{array}$} \\
\hline $\mathrm{F}$ & 97 & $23(23.7)$ & $13(13.4)$ & $22(22.7)$ & $31(32.0)$ & $8(8.2)$ & \\
\hline ICLT -ve, M & 67 & $13(\mathbf{1 9 . 4})^{\mathrm{b}}$ & $14(20.9)$ & $22(32.9)^{\mathrm{a}}$ & $9(13.4)$ & $9(13.4)$ & \multirow{2}{*}{$\begin{array}{l}x^{2}=6.32 \\
p=0.18\end{array}$} \\
\hline $\mathrm{F}$ & 228 & $73(32.1)^{b}$ & $55(24.1)$ & $49(21.5)^{\mathrm{a}}$ & $27(11.8)$ & $24(10.5)$ & \\
\hline \multicolumn{8}{|l|}{ Echotexture and ICLT } \\
\hline Hypoechoic, ICLT + ve & 58 & $8(13.8)$ & $6(10.3)$ & $14(24.1)$ & $23(39.7)^{\mathrm{a}}$ & $7(12.1)$ & \multirow{2}{*}{$\begin{array}{l}x^{2}=5.77 \\
p=0.22\end{array}$} \\
\hline ICLT-ve & 82 & $15(18.3)$ & $16(19.5)$ & $15(18.3)$ & $21(25.6)^{\mathrm{a}}$ & $15(18.3)$ & \\
\hline Isooechoic, ICLT + ve & 55 & $17(30.9)$ & $11(20.0)$ & $13(23.6)$ & $11(\mathbf{2 0 . 0})^{\mathrm{b}}$ & $3(5.5)$ & \multirow{2}{*}{$\begin{array}{l}X^{2}=8.70 \\
p=0.069\end{array}$} \\
\hline ICLT-ve & 213 & $71(33.3)$ & $53(24.9)$ & $56(26.3)$ & $15(7.0)^{b}$ & $18(8.5)$ & \\
\hline \multicolumn{8}{|l|}{ Echotext, ICLT and sex } \\
\hline Hypoechoic, ICLT + ve, M & 9 & 0 & $2(22.2)$ & $4(44.4)$ & $1(11.1)^{\mathrm{a}}$ & $2(22.2)$ & \multirow{2}{*}{$\begin{array}{l}X^{2}=7.84 \\
p=0.097\end{array}$} \\
\hline $\mathrm{F}$ & 49 & $8(16.3)$ & $4(8.2)$ & $10(20.4)$ & $22(44.9)^{\mathrm{a}}$ & $5(10.2)$ & \\
\hline Isoechoic, ICLT + ve, M & 7 & $2(28.6)$ & $2(28.6)$ & $1(14.2)$ & $2(28.6)$ & 0 & \multirow{2}{*}{$\begin{array}{l}x^{2}=1.34 \\
p=0.85\end{array}$} \\
\hline $\mathrm{F}$ & 48 & $15(31.2)$ & $9(18.8)$ & $12(25.0)$ & $9(18.8)$ & $3(6.2)$ & \\
\hline Hypoechoic, ICLT-ve, M & 15 & $0^{\mathrm{b}}$ & $5(33.3)$ & $2(13.3)$ & $5(33.3)$ & $3(20.0)$ & \multirow{2}{*}{$\begin{array}{l}x^{2}=5.86 \\
p=0.21\end{array}$} \\
\hline $\mathrm{F}$ & 67 & $15(22.4)^{b}$ & $11(16.4)$ & $13(19.4)$ & $16(23.9)$ & $12(17.9)$ & \\
\hline Isoechoic, ICLT-ve, M & 52 & $13(25.0)$ & $9(17.3)$ & $20(38.5)^{b}$ & $4(7.7)$ & $6(11.5)$ & \multirow{2}{*}{$\begin{array}{l}x^{2}=7.71 \\
p=0.10\end{array}$} \\
\hline $\mathrm{F}$ & 161 & $58(36.0)$ & $44(27.3)$ & $36(22.4)^{b}$ & $11(6.8)$ & $12(7.5)$ & \\
\hline
\end{tabular}

Equivalence between the SIAPEC 2014 classification and the Bethesda classification: TIR2: Benign; TIR3A: Atypia of undetermined significance or follicular lesion of undetermined significance (AUS/FLUS); TIR3B: Follicular neoplasm or suspicious for a follicular neoplasm (FN/SN); TIR4: Suspicious for malignancy; TIR5: Malignant. Raw percentages are shown in parentheses. Comparison between two proportions within each category: a) p between 0.10 and 0.05, b) $<0.05$, c) $p<0.01$, d) $p<0.001$, e) $p<0.0001$. 

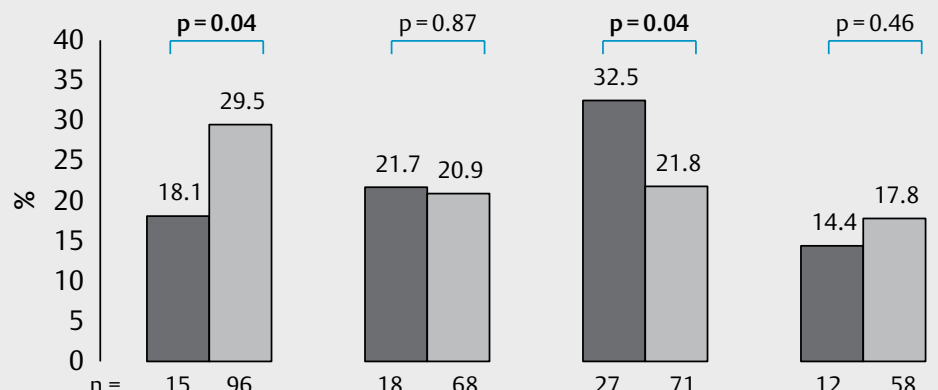

$12 \quad 58$
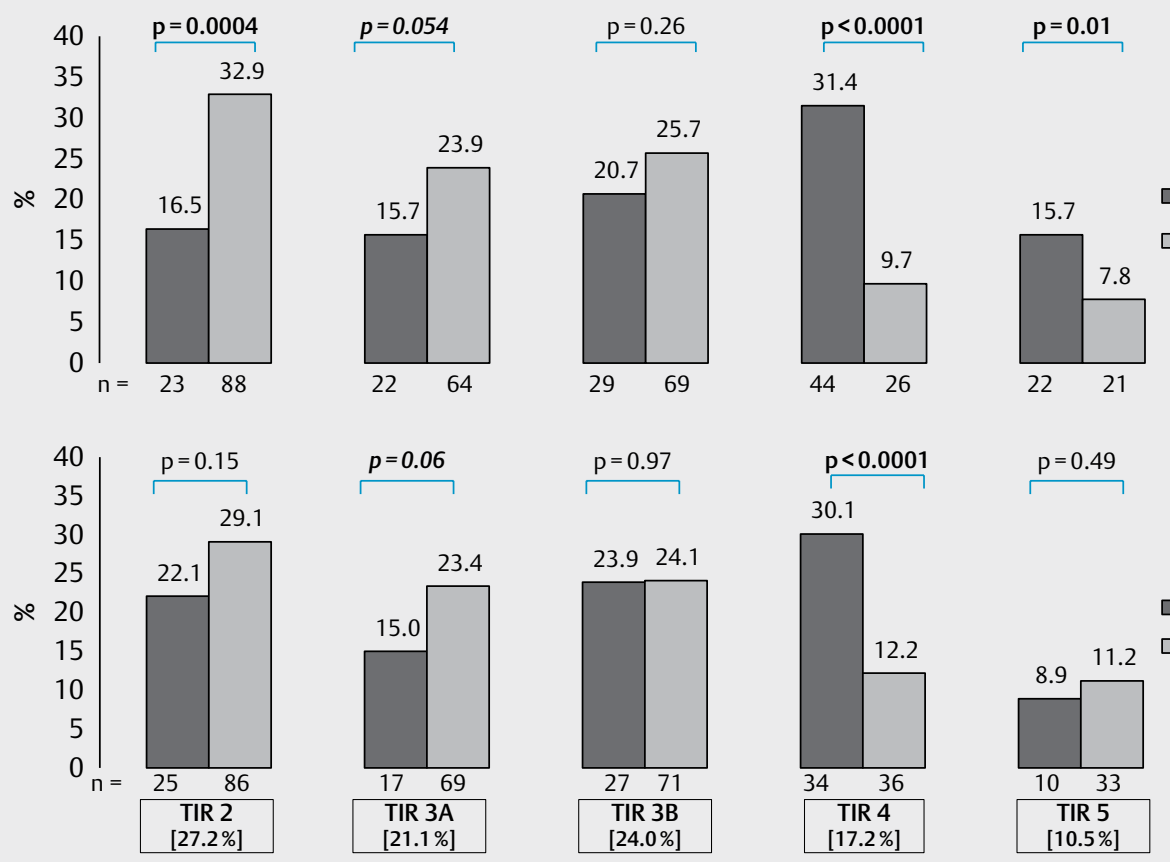

$$
\begin{array}{ll}
\square \text { HYPOECHOIC } & (n=140) \chi^{2}=43.7 \\
\square \text { ISOECHOIC } & (n=268) P=7.4 \times 10^{9}
\end{array}
$$

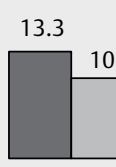

$\square$ MALES $\quad(n=83) \chi^{2}=7.53$

$\square$ FEMALES $(n=325) P=0.11$

1132
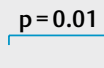

abundant in the TIR2 through TIR3B categories (20.7-29.6\%) than in the TIR4 $(\mathrm{p}<0.001)$ or TIR 5 categories $(51.2 \%, \mathrm{p}<0.01)$.

The distribution of the five cytological classes also differed significantly in the ICLT + ve nodules compared to the ICLT-ve nodules $(p=0.0005$; $>$ Fig. 1 , bottom panel), such difference being accounted particularly by the TIR4 class [34/113 (30.1\%) vs. 36/295 $\left.(12.2 \%), X^{2}=18.4, p=1.8 \times 10^{-5}, \mathrm{OR}=3.1(1.8-5.3)\right] . \vee$ Figure 2 (bottom panel) shows that ICLT was present in $19.8-27.6 \%$ of nodules in all cytological classes except the TIR4 class, each percentage being significantly lower $(p<0.005)$ than the $48.6 \%$ observed in the TIR 4 class.

In $>$ Table 2, data are presented based on combinations of any two or all three indices (gender, echotexture and ICLT). If nodules were separated based on echotexture, then the distribution of the isoechoic nodules among the five FNA classes was statistically similar in males compared to females $(p=0.24)$, while the distribution of the hypoechoic nodules was borderline significantly different in males compared to females $(p=0.055)$. If nodules were separated based on presence/absence of ICLT, then the distribution of ICLT + ve nodules did not differ significantly in males compared to females ( $p=0.48)$, and this also applied to the ICLT-ve nodules $\left(d f=4, X^{2}=6.32, p=0.18\right)$. If stratification was based on ICLT and echotexture combined, then the distribution of the ICLT + ve and hypoechoic nodules was borderline significantly different in males compared to females $(p=0.097)$, but it was insignificant for $I C L T+$ ve and isoechoic nodules ( $\left.d f=4, x^{2}=1.34, p=0.85\right)$, for ICLTve and hypoechoic nodules $(p=0.21)$, and it was just borderline significantly different for ICLT-ve and isoechoic nodules $(p=0.10)$ $(\triangleright$ Table 2).

\section{Frequency of malignancy taking into account gender, echotexture, and ICLT}

Data are summarized in $\mathbf{F i g}$. 3. The overall rate of histologically verified malignancy was $145 / 408$ (35.5\%), with an insignificantly greater risk for males compared to females ( 42.2 vs. $33.8 \%$, $p=0.16$ ). As expected, the frequency of histological malignancy was greater in the hypoechoic nodules compared to the isoechoic nodules ( 55.7 vs. $\left.25.0 \%, p=7.6 \times 10^{-10}\right)$. Such frequency was also greater in ICLT + ve nodules compared to the ICLT-ve ones ( $48.7 \mathrm{vs.}$ $30.5 \%, p=0.0006$; Fig. 3a). 


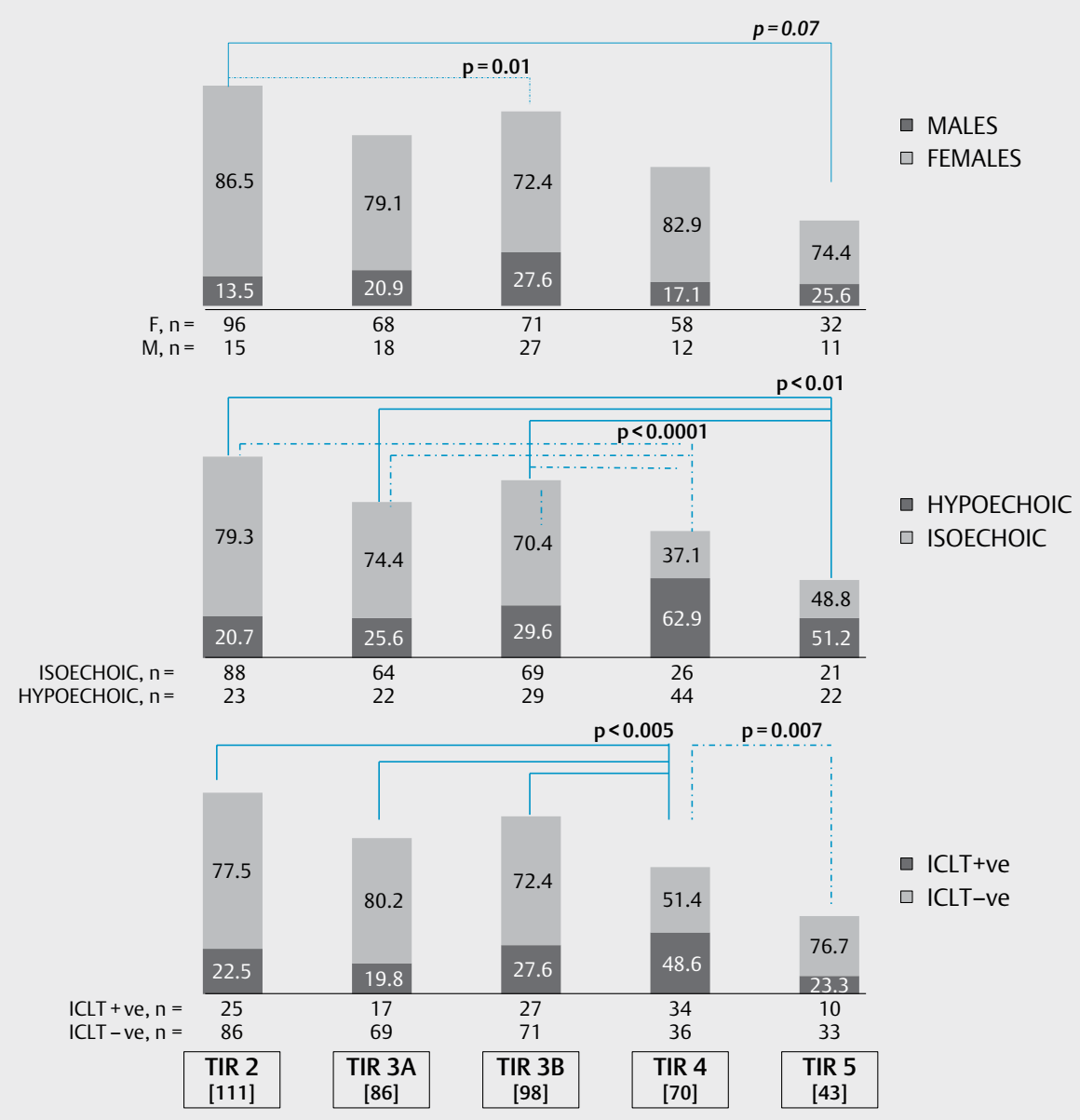

- Fig. 2 Percentage in each cytological class of risk according to gender, nodular echogencity, and ICLT status. The percentages of given indices are shown in the columns.

Considering the combined characteristics "echotexture and gender", the two genders had a very similar frequency of malignancy within the hypoechoic nodules (58.3\% for males vs. $55.2 \%$ for females, $p=0.78$ ), whereas males had a frequency of malignancy significantly greater than females within the isoechoic nodules ( 35.5 vs. $22.2 \%, p=0.033$ ). In turn, the difference between $58.3 \%$ (hypoechoic) and $35.5 \%$ (isoechoic) in males was borderline significant ( $p=0.057$ ), while the equivalent difference in females was highly significant (55.2 vs. $22.2 \%, p<0.0001$; $>$ Fig. $3 a$ ).

Considering the combined characteristics "ICLT status and gender", the frequency of malignancy was insignificantly greater in females compared to males within the ICLT + ve nodules ( $50.5 \mathrm{vs.} 37.5 \%$, $\mathrm{p}=0.33$ ) but it was significantly greater in males compared to females within the ICLT-ve nodules ( 43.3 vs. $26.7 \%, p=0.0098$ ). In turn, the difference between $37.5 \%$ (ICLT + ve) and $43.3 \%$ (ICLT-ve) in males was insignificant, whereas the equivalent difference in females was highly significant ( 50.5 vs. $26.7 \%$, p < 0.0001) ( Fig. 3a).

Considering the combined characteristics "echotexture and ICLT status" ( $\triangleright$ Fig. $\mathbf{3 b}$ ), the frequency of malignancy was consistently greater in the ICLT + ve compared to the ICLT-ve nodules, but at least borderline significantly only in the hypoechoic nodules $(63.8$ vs. $50.0 \%, p=0.10)$. Hypoechogenicity was consistently associat- ed with a greater risk of malignancy compared to isoechogeneicity, both in the ICLT + ve nodules ( 63.8 vs. $32.7 \%, p=0.001)$ and in the ICLT-ve nodules ( 50.0 vs. $23.0 \%$, $p<0.0001$ ).

Considering the three characteristics altogether, the greatest frequencies of malignancy was observed in hypoechoic, ICLT + ve nodules of females (67.3\%) and in hypoechoic, ICLT-ve nodules of males (66.7\%), and the lowest in isoechoic, ICLT-ve nodules of females (18.6\%; Fig. 3b).

\section{Frequency of malignancy taking into account gender, echotexture, ICLT, and cytological category}

Data are summarized in $\triangleright$ Table 3. According to the cytological classes of risk, ranged from $2.7 \%$ for TIR2 nodules to $100 \%$ for TIR5 nodules. Except for the invariable $100 \%$ rate in the TIR5 category, rates of malignancy varied greatly within each of the remaining four classes (0-25\% for TIR2, 0-22.2\% for TIR3A, 0-100\% for TIR3B, and 63.6-100\% for TIR4). Disregarding the TIR5 category and other categories where the denominator was less than 5 , a $100 \%$ rate of malignancy was detected only in the TIR4 hypoechoic nodules of males, the scanty size of cases of TIR4/hypoechoic/ICLT + ve precluding the possibility of ascertaining whether the presence of ICLT made a difference in TIR4 hypoechoic nodules of males. In the TIR3B 
a

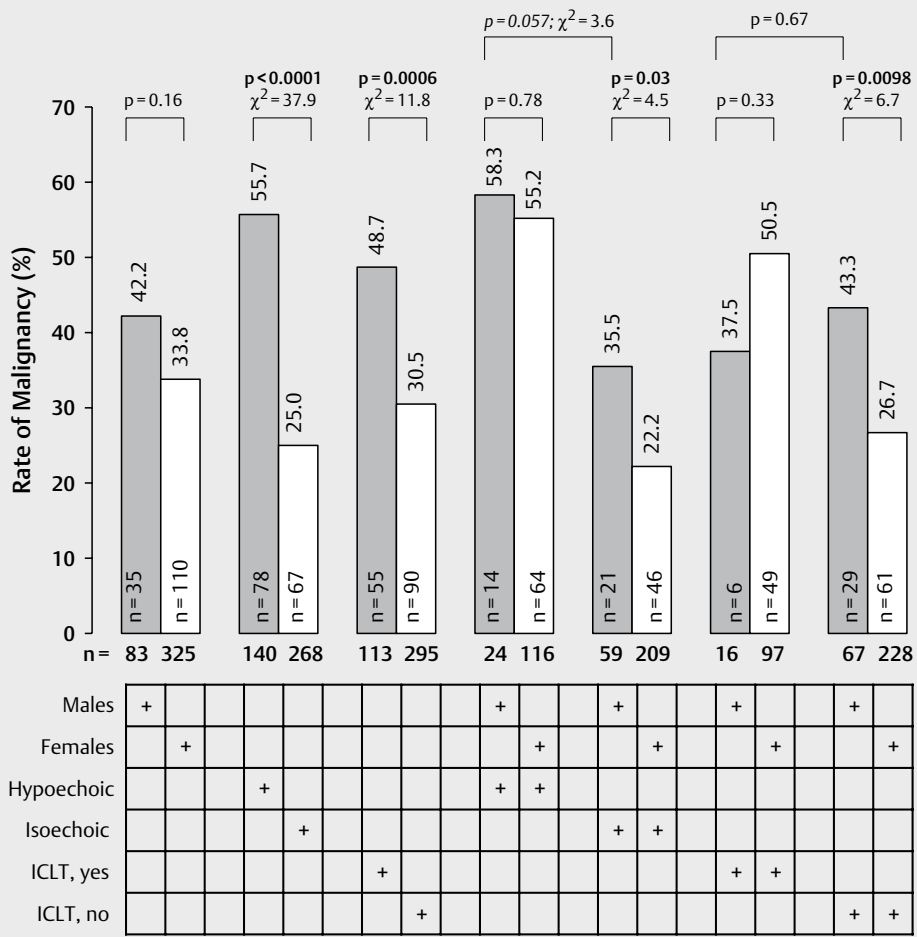

b

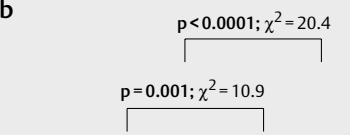

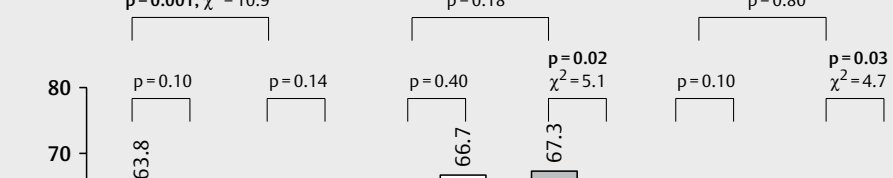

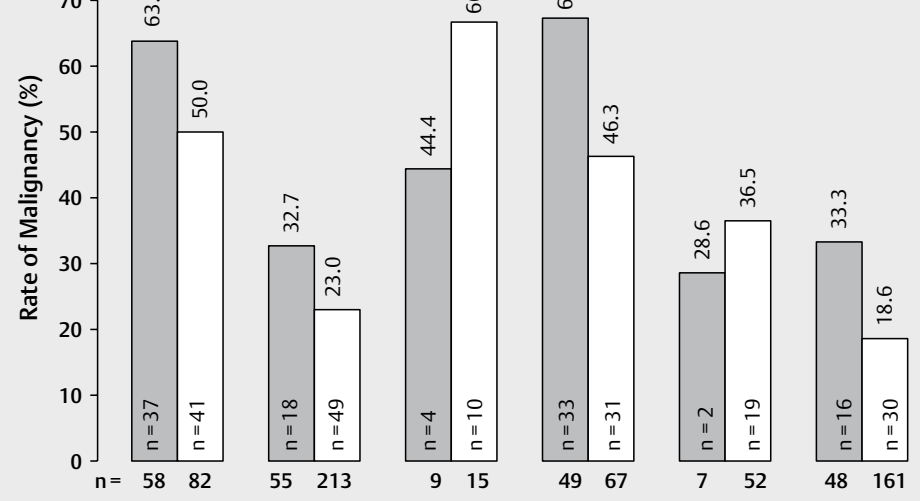

\begin{tabular}{|c|c|c|c|c|c|c|c|c|c|c|c|c|}
\hline Males & & & & & + & + & & & + & + & & \\
\hline Females & & & & & & & + & + & & & + & + \\
\hline Hypoechoic & + & + & & & + & + & + & + & & & & \\
\hline Isoechoic & & & + & + & & & & & + & + & + & + \\
\hline ICLT, yes & + & & + & & + & & + & & + & & + & \\
\hline ICLT, no & & + & & + & & + & & + & & + & & + \\
\hline
\end{tabular}

Fig. 3 a and b: Rate of malignancy according to the given indices. a: comparison of nodular echotexture or ICLT status singly considered with gender. b: comparison of nodular echotexture and ICLT status regardless of (on the left) or taking into account (on the right) gender. 
- Table 3 Frequency of histologically verified malignancy in the cytological classes based on given indices.

\begin{tabular}{|c|c|c|c|c|c|c|}
\hline & \multicolumn{5}{|c|}{ Cytological Classes of risk (No. and \%) } & \multirow[b]{2}{*}{ Statistics } \\
\hline & TIR2 & TIR3A & TIR3B & TIR4 & TIR5 & \\
\hline All & $3 / 111(2.7)$ & $4 / 86(4.6)$ & $38 / 98(38.8)$ & $57 / 70(81.4)$ & $43 / 43(100)$ & \\
\hline \multicolumn{7}{|l|}{ Echotext, ICLT and sex } \\
\hline Hypoechoic, ICLT + ve, M (n=4) & 0 & $0 / 2$ & $1 / 4(25.0)^{\mathrm{a}}$ & $1 / 1(100)$ & $2 / 2(100)$ & \multirow{2}{*}{$\begin{array}{l}X^{2}=36.6 \\
\mathbf{p}=\mathbf{0 . 0 0 0 3}\end{array}$} \\
\hline$F(n=33)$ & $2 / 8(25.0)$ & $0 / 4$ & $8 / 10(80.0)^{\mathrm{a}}$ & $18 / 22(81.8)$ & $5 / 5(100)$ & \\
\hline Isoechoic, ICLT + ve, M (n=2) & $0 / 2$ & $0 / 2$ & $0 / 1$ & $2 / 2(100)$ & 0 & \multirow{2}{*}{$\begin{array}{l}X^{2}=33.0 \\
\mathbf{p}=\mathbf{0 . 0 0 1}\end{array}$} \\
\hline$F(n=16)$ & $0 / 15$ & $2 / 9(22.2)$ & $4 / 12(33.3)$ & $7 / 9(77.8)$ & $3 / 3(100)$ & \\
\hline Hypoechoic, ICLT-ve, M $(n=10)$ & 0 & $0 / 5$ & $2 / 2(100)$ & $5 / 5(100)$ & $3 / 3(100)$ & \multirow{2}{*}{$\begin{array}{l}x^{2}=76.5 \\
p<0.0001\end{array}$} \\
\hline$F(n=31)$ & $0 / 15$ & $0 / 11$ & $5 / 13(38.5)$ & $14 / 16(87.5)$ & $12 / 12(100)$ & \\
\hline Isoechoic, ICLT-ve, M ( $n=19)$ & $0 / 13$ & $1 / 9(11.1)$ & $9 / 20(45.0)$ & $3 / 4(75.0)$ & $6 / 6(100)$ & \multirow{2}{*}{$\begin{array}{l}x^{2}=115 \\
p<0.0001\end{array}$} \\
\hline$F(n=31)$ & $1 / 58(1.7)$ & $1 / 44(2.3)$ & $9 / 36(25.0)$ & $7 / 11$ (63.6) & $12 / 12(100)$ & \\
\hline \multicolumn{7}{|c|}{$\begin{array}{l}\text { Equivalence between the SIAPEC } 2014 \text { classification and the Bethesda classification: TIR2: Benign; TIR3A: Atypia of undetermined significance or } \\
\text { follicular lesion of undetermined significance (AUS/FLUS); TIR3B: Follicular neoplasm or suspicious for a follicular neoplasm (FN/SN); TIR4: Suspicious } \\
\text { for malignancy; TIR5: Malignant. The gray background indicates percentages of malignancy greater than these thresholds: } 3 \% \text { (TIR2), } 10 \% \text { (TIR3A), } \\
30 \% \text { (TIR3B), } 80 \% \text { (TIR4). Comparison between two proportions: a) p between } 0.10 \text { and } 0.05, b) p<0.05, c) p<0.01, d) p<0.001, e \text { ) p }<0.0001 \text {. } \\
\text { Numbers in parentheses are percentages. Degree of freedom (df) is } 12 \text {. }\end{array}$} \\
\hline
\end{tabular}

category, a rate over 2 -fold greater than the $30 \%$ threshold of malignancy was detected for hypoechoic/ICLT + ve nodules of females ( $80 \%$ ), which contrasts sharply with the $38.5 \%$ rate of malignancy detected for hypoechoic/ICLT-ve nodules of females. In the TIR3A category, a rate over 2 -fold greater than the $10 \%$ threshold of malignancy was detected for isoechoic/ICLT + ve nodules of females ( $22.2 \%)$, which contrasts sharply with the $2.3 \%$ rate of malignancy detected for isoechoic/ICLT-ve nodules of females. In the TIR2 category, a rate over 2 -fold greater than the $3 \%$ threshold of malignancy was detected for hypoechoic/ICLT + ve nodules of females (25\%).

Also noteworthy are, at the other extreme, the said low rates of malignancy in the TIR3B category (38.5\% for hypoechoic/ICLT-ve nodules of females) and TIR3A (2.3\% for isoechoic/ICLT-ve nodules of females). No malignancy at all was observed in these nodules: for the TIR3A in those hypoechoic regardless of ICLT and sex; for the TIR2 in those of males regardless of echotexture and ICLT, and in isoechoic/ICLT + ve and hypoechoic/ICLT-ve of females.

\section{Summary}

Taking into account only the echotexture of a thyroid nodule prior to FNA, its risk of malignancy in males compared to females is similar when the nodule is hypoechoic ( 58.3 vs. $55.2 \%, p=0.78$ ), but greater when the nodule is isoechoic ( 35.5 vs. $22.2 \%, p=0.03$ ). Taking into account only the presence or absence of ICLT prior to FNA, the risk of malignancy is greater in males when ICLT is absent (43.3 vs. $26.7 \%, p=0.01$ ), but insignificantly lower than females when ICLT is present ( 37.5 vs. $50.5 \%, p=0.33$ ). Taking into account both echotexture and ICLT prior to FNA, there is only one situation where the risk of malignancy is statistically different in one gender compared to the other, males having a greater risk: the isoechoic/ ICLT + ve nodule (36.5 vs. $18.6 \%, p=0.008)$.

There is a payoff if the cytologist complements the FNA report with the presence or absence of ICLT and the clinician interprets the report in the background of echogenicity of the interrogated nodule and gender. This advantage is very evident in the TIR3B category. Given two males both having a hypoechoic nodule worthy of FNA, the risk of malignancy is enormously different. Indeed, this risk is $25 \%$ in the man with an ICLT + ve nodule, but it is $100 \%$ in the man with an ICLT-ve nodule. The gap is smaller, but clinically relevant, if the punctured nodule in another two men is isoechoic. Indeed, the ICLT + ve nodule of the first man has no risk at all to be malignant, whereas the ICLT-ve nodule of the second man has a $45 \%$ risk. In females, the advantage is limited to the hypoechoic nodules and the correlation with ICLT goes in the opposite direction compared to men. Thus, women with an ICLT + ve hypoeochoic nodule have an $80 \%$ risk of malignancy as compared to $38.5 \%$ of women with an ICLT-ve hypoeochoic nodule.

The stratification of the four categories spanning TIR2 through TIR4 [3,5] (benign through suspicious for malignancy) taking into account ICLT, echotexture (considering only hypoechogenous and isoechogenous nodules) and gender leads to formation of 16 subgroups in males and 16 in females. This stratification is more useful in males than in females, since it leads to identification of 4 subgroups with $100 \%$ risk of malignancy each and another 8 subgroups with zero \% risk. By comparison, none of the 16 subgroups in females has a $100 \%$ risk, and only 4 have zero \% risk. Within the 8 subgroups of the TIR3A and TIR3B categories (AUS/FLUS and FN/SFN) in males, 1 has a $100 \%$ risk of malignancy and 4 have zero \% such risk. By comparison, none of the 8 subgroups has $100 \%$ risk of malignancy and only 2 have zero \% such risk. 


\section{Discussion}

As mentioned in the Introduction, in the same cohort of patients of the present study, we have previously found that the presence/ absence of ICLT is associated with some sexually dimorphic characteristics of thyroid nodules [7]. One previous finding was that ICLT + ve hypoechoic nodules of females and ICLT-ve hypoechoic nodules of males had the greatest rate of histologically verified malignancy (67\% both), while ICLT-ve isoechoic nodules of females had the lowest (19\%). In the present study, we have expanded our investigations. We added the cytological classification to other two indices of the thyroid nodule (ICLT, echotexture) and to the patient's gender, with the practical goal of assessing whether the combined value of these four preoperative factors would have maximized predictivity of the nature (benign or malignant) of the nodule itself.

Here we have shown that the risk of malignancy of thyroid nodules is modulated by gender, echotexture and ICLT status. Particularly, after the nodules have been interrogated by FNA, we have shown that the distribution of the five cytologically adequate categories also depends from echotexture (hypoechoic vs isoechoic), ICLT (presence vs absence), and gender. We have also shown that, in each of the first four categories [TIR2 (benign) though TIR4 (suspicious for malignancy)], these three variables impact on the risk for malignancy. For instance, in the regard to the first point, FNA of a hypoechoic nodule with absent ICLT has a $22 \%$ chance of being TIR2 in a woman but $0 \%$ chance of being so in a man. Because ICLT can be detected only at cytological reading, for pre-FNA predictivity of the cytological category, ICLT has to be disregarded. Thus, based on echotexture and gender, a hypoechoic nodule has a $0 \%$ chance of being TIR2 and $21 \%$ chance of being TIR5 in a man, but corresponding chances of 20 and $15 \%$ in a woman.

Much more relevant is the second point, namely once a FNA category has been assigned, what is the chance of true malignancy? Omitting the TIR5 category because of the invariable $100 \%$ rate of malignancy, the approximate $3,5,39$, and $81 \%$ risk of malignancy for the TIR2, TIR3A, TIR3B and TIR4 cannot be applied uniformly for all FNA-interrogated nodules. For instance, males with a TIR2 nodule have $0 \%$ chance that this nodule is malignant. In females, such chance for a TIR2 is absent or minimal (0-1\%), except when the nodule is hypoechoic and ICLT + ve (25\%). At the other extreme (TIR4), males have a $100 \%$ chance of malignancy except when the nodule is isoechoic and ICLT-ve (75\%). In females, the malignancy rate for a TIR4 nodule is $<90 \%$, with a range from $87 \%$ (hypoechoic and ICLT-ve) to $64 \%$ (isoechoic and ICLT-ve). Worthy of note is analysis of this risk in the so-called gray area nodules (TIR3A and TIR3B). For the TIR3A category, the risk is restricted to isoechoic nodules [ICLT + ve in women (22\%) and ICLT-ve in men (11\%)]. This $22 \%$ risk for a TIR3A isoechoic/ICLT + ve nodule as well as the said $25 \%$ risk for a TIR2 hypoechoic/ICLT + ve nodule, both detected among women, fall within the $15-30 \%$ range for a TIR3B nodule ( $\triangleright$ Table 1), implying that these two subgroups of patients should receive the same recommendations as for the TIR3 nodules [ "surgical exeresis" based on SIAPEC2014 system [3] and "Molecular testing, lobectomy" based on Bethesda system [5]\}. Recommendations for a TIR2 nodule are "clinical and sonographic follow-up" $[3,5]$, while those for a TIR3A nodule are "repeat FNA or follow-up"
[3] and "repeat FNA, molecular testing, or lobectomy" [5]. For the TIR3B nodules, outliers with risk of more advanced categories are women with a hypoechoic/ICLT + ve nodule $(80 \%)$ and men with a hypoechoic/ICLT-ve nodule (100\%).

Our data in the two gray area categories have implications for the issue of molecular testing. There were $184 / 408$ nodules (184/408 patients) in the TIR3A ( $n=86 ; 18 M, 68 \mathrm{~F})$ or TIR3B ( $n=98$; $27 \mathrm{M}, 71 \mathrm{~F}$ ) categories, accounting for almost half $(45.1 \%)$ of the whole cohort. In lieu of universal molecular testing (that is, all 184 nodules), testing can be spared to those with zero \% risk of malignancy and those with $>60 \%$ risk of malignancy. These are 24 nodules in the TIR3A category [ $27.9 \%$ of $86 ; 9 / 18 \mathrm{M} \mathrm{(50 \% )} \mathrm{and} 15 / 68 \mathrm{~F}$ (23.4\%)] and 12 nodules in the TIR3B category [12.2\%; 2/27 M (7.5\%) and 10/71 F (14.1\%)]. Thus, molecular testing can be spared to one in four TIR3A nodules and one in eight TIR3B nodules. As a novel ramification of our data, molecular testing can be considered in a small fraction of women with TIR2 hypoechoic/ICLT + ve nodules (8/96 in our cohort), because this subgroup carries a $25 \%$ chance of malignancy.

One strength of this study is the homogeneity ensured by US, FNA and reading, as all of them were performed by the same operator. Another strength is the homogeneity ensured by all patients coming from a relatively narrow geographic area, which is the Syracuse province, in southeastern Sicily. Furthermore, our data on the widely different risks for malignancy within the same FNA category may help to explain the different data from the literature on molecular testing to exclude or to indicate malignancy $[2,13,14]$. Indeed, depending on the relative abundance of subgroups (based on gender, ICLT and echotexture) in the TIR3A (AUS/FLUS) and TIR3B (FN/SFN) nodules that are interrogated by molecular biology testing, different negative predictive values (or positive predictive values) will be obtained. One limitation of our study is the smaller size of the male group compared to the female group, though it is known that thyroid diseases prevail in females. Another limitation is the lack of hyperechoic nodules in our cohort.

In summary, upon operating within the TIR2 through TIR4 categories (benign through suspicious for malignancy) a gender-specific stratification of hypoechoic and isoechoic thyroid nodules that takes into account the absence or presence of ICLT, one can maximize in the malignancy (or benignity) direction the average predictivity of that given category. This operation identifies a greater number of subgroups having extreme risks of malignancy ( $0 \%$ and $100 \%$ in the male gender. Our findings are expected to impact on future FNA-driven guidelines for the management of the thyroid nodules.

\section{Acknowledgements}

The authors thank Dr. Elisabetta Papalia for her contribution in data storage.

\section{Conflict of Interest}

The authors declare that they have no conflict of interest. 


\section{References}

[1] Francis GL, Waguespack SG, Bauer A] et al. American Thyroid Association Guidelines Task Force. Management guidelines for children with thyroid nodules and differentiated thyroid cancer. Thyroid 2015; 25: 716-759

[2] Haugen BR, Alexander EK, Bible KC et al. American Thyroid Association Management guidelines for adult patients with thyroid nodules and differentiated thyroid cancer: The American Thyroid Association Guidelines Task Force on Thyroid nodules and differentiated thyroid cancer. Thyroid 2016; 26: 1-133

[3] Nardi F, Basolo F, Crescenzi A et al. Italian consensus for the classification and reporting of thyroid cytology. J Endocrinol Invest 2014; 37: 593-599

[4] Cibas ES, Ali SZ. The Bethesda system for reporting thyroid cytopathology. Thyroid 2009; 19: 1159-1165

[5] Cibas ES, Ali SZ. The 2017 Bethesda system for reporting thyroid cytopathology. Thyroid 2017; 27: 1341-1346

[6] Nikiforov YE, Seethala RR, Tallini G et al. Nomenclature revision for encapsulated follicular variant of papillary thyroid carcinoma: A paradigm shift to reduce overtreatment of indolent tumors. JAMA Oncol 2016; 2: 1023-1029

[7] Arena S, Benvenga S. Gender-specific correlation of intranodular chronic lymphocytic thyroiditis with thyroid nodule size, echogenicity, and histologically-verified cytological class of malignancy risk. J Clin Transl Endocrinol 2018; 14: 39-45
[8] Cooper DS, Doherty GM, Haugen BR et al. American Thyroid Association (ATA) guidelines taskforce on thyroid nodules and differentiated thyroid cancer, revised american thyroid association management guidelines for patients with thyroid nodules and differentiated thyroid cancer. Thyroid 2009; 19: 1167-1214

[9] Rizzo M, Rossi RT, Bonaffini $O$ et al. Increased annual frequency of Hashimoto's thyroiditis between years 1988 and 2007 at a cytological unit of Sicily. Ann Endocrinol (Paris) 2010; 71: 525-534

[10] Arena S, Latina A, Baratta R et al. Chronic lymphocytic thyroiditis: Could it be influenced by a petrochemical complex? Data from a cytological study in South-Eastern Sicily. Eur ] Endocrinol 2015; 172: 383-389

[11] Bhatia A, Rajwanshi A, Dash R] et al. Lymphocytic thyroiditis - is cytological grading significant? A correlation of grades with clinical, biochemical, ultrasonographic and radionuclide parameters. CytoJournal 2007; 30: 41-46

[12] Baloch ZW, LiVolsi VA, Asa SL et al. Diagnostic terminology and morphologic criteria for cytologic diagnosis of thyroid lesions: a synopsis of the National Cancer Institute thyroid fine-needle aspiration state of the Science Conference. Diagnost Cytopathol 2008; 36: 425-437

[13] Sahli ZT, Smith PW, Umbricht CB et al. Preoperative molecular markers in thyroid nodules. Front Endocrinol (Lausanne) 2018; 9: 179

[14] Roth MY, Witt RL, Steward DL. Molecular testing for thyroid nodules: Review and current state. Cancer 2018; 124: 888-898 